\title{
Dependence of Hurricane Intensity and Structures on Vertical Resolution and Time-Step Size
}

\author{
Da-Lin ZHANG* and Xiaoxue WANG \\ Department of Meteorology, University of Maryland, College Park, Maryland, 20742 USA
}

(Received 24 January 2003; revised 6 May 2003)

\begin{abstract}
In view of the growing interests in the explicit modeling of clouds and precipitation, the effects of varying vertical resolution and time-step sizes on the 72-h explicit simulation of Hurricane Andrew (1992) are studied using the Pennsylvania State University/National Center for Atmospheric Research (PSU/NCAR) mesoscale model (i.e., MM5) with the finest grid size of $6 \mathrm{~km}$. It is shown that changing vertical resolution and time-step size has significant effects on hurricane intensity and inner-core cloud/precipitation, but little impact on the hurricane track. In general, increasing vertical resolution tends to produce a deeper storm with lower central pressure and stronger three-dimensional winds, and more precipitation. Similar effects, but to a less extent, occur when the time-step size is reduced. It is found that increasing the low-level vertical resolution is more efficient in intensifying a hurricane, whereas changing the upper-level vertical resolution has little impact on the hurricane intensity. Moreover, the use of a thicker surface layer tends to produce higher maximum surface winds. It is concluded that the use of higher vertical resolution, a thin surface layer, and smaller time-step sizes, along with higher horizontal resolution, is desirable to model more realistically the intensity and inner-core structures and evolution of tropical storms as well as the other convectively driven weather systems.
\end{abstract}

Key words: hurricane intensity, vertical resolution, numerical weather prediction

\section{Introduction}

Rapid growth in computing power has recently allowed us to use small horizontal grid lengths, even down to $1-2 \mathrm{~km}$, to model the inner structures and evolution of various mesoscale convective systems (MCSs). Indeed, increasing horizontal resolution with better model physical parameterizations has shown significant improvements in the quality of numerical weather prediction (NWP). However, the adequacy of vertical resolution in the current NWP models has recently been questioned, and some studies have indicated that increasing horizontal resolution alone does not always guarantee a better solution, particularly in the presence of phase changes.

For example, Lindzen and Fox-Rabinovitz (1989) derived a consistency criterion between horizontal $(\triangle X)$ and vertical $(\triangle Z)$ resolution for quasigeostrophic flows, which is given by

$$
\triangle Z=\frac{f}{N} \triangle X
$$

where $f$ is the Coriolis parameter and $N$ is the buoyancy frequency. It is apparent from Eq. (1) that vertical and horizontal resolutions should be proportional to each other, and a finer vertical resolution should be used for the tropics or for a more stable fluid. They pointed out that a fine horizontal resolution, without considering an appropriate vertical resolution, would lead to the production of 'noisy' fields and may degrade the overall accuracy of the solution. They argued that the current NWP models, despite their inconsistent horizontal and vertical resolutions, could still produce reasonable results because of the use of too strong smoothing and damping.

Based on a two-dimensional hydrostatic primitive equation model, Pecnick and Keyser (1989) derived a relationship that physically relates horizontal scales to vertical scales of an upper-level frontal structure, in which frontogenesis is forced solely by confluence. That is, for a given horizontal resolution, there is an optimal thickness of the vertical layer,

$$
\triangle Z=m \triangle X
$$

*E-mail: dalin@atmos.umd.edu 
where $\triangle Z$ is the optimal vertical grid spacing in pseudo-height coordinates, and $m$ is the frontal slope. After analyzing the time series of various variables (e.g., along-frontal velocity, absolute vorticity, vertical motion, and horizontal potential temperature $\theta$ gradients), they concluded that (a) increasing both the horizontal and vertical resolutions results in better frontal structures; and (b) inconsistent horizontal and vertical resolutions tend to generate spurious wavelike features superposed on the frontal zone.

In contrast to the above results that were obtained with dry dynamics equations, Persson and Warner (1991) studied the resolution consistency in a hydrostatic (moist) simulation of conditional symmetric instability associated with frontal rainbands. They noted the development of spurious gravity waves when criterion (2) is not satisfied. Moreover, the wave amplitudes increase as the vertical resolution decreases.

Similar studies have also been performed to examine the importance of the model resolution consistency in simulating, for example, middle atmospheric circulations with a climate model (Hamilton et al., 1999), heat transport with an oceanic general circulation model (Weaver and Sarachik, 1990), and cloud and radiation parameterizations in a climate model (Lane et al., 2000). All of the above-mentioned studies suggested that one should not simply increase the horizontal resolution without considering appropriate vertical resolution. In addition, these studies indicated that a consistent model resolution would lead to more realistic simulations and eliminate some artificial features and noises, such as spurious gravity waves. However, few studies have been conducted to examine the significance of changing vertical resolution in simulating tropical cyclones in which tremendous latent heat release occurs.

Thus, one of the objectives of this study is to examine the sensitivity of the explicit simulation of Hurricane Andrew (1992) to varying vertical resolutions in terms of its intensity and inner-core structures. Liu et al. (1997; 1999) have shown a 72-h successful simulation of the hurricane track and intensity, as well as the structures of the eye, the eyewall, spiral rainbands, the radius of maximum winds (RMW), and other innercore features as compared to available observations and the results of previous hurricane studies. In this study, the model set-ups, such as the model domains, grid sizes, initial conditions, and physics options, are the same as those used by Liu et al. (1997), except for the vertical resolution. The next section describes briefly the numerical model used for this study and the case study. Sections 3 and 4 present the experimental design and sensitivity simulations, respectively. A summary and conclusions are given in the final section.

\section{Model description and case study}

In the present study, a two-way interactive, movable, triply-nested, cloud-resolving, nonhydrostatic version of the Pennsylvania State University/National Center for Atmospheric Research (PSU/NCAR) mesoscale model (i.e., MM5; see Dudhia, 1993) is used. MM5 uses the vertical $\sigma$ coordinates defined as

$$
\sigma=\frac{p-p_{\mathrm{t}}}{p_{\mathrm{s}}-p_{\mathrm{t}}}
$$

where $p_{\mathrm{s}}$ is the model bottom pressure at the initial time, and $p_{\mathrm{t}}(=50 \mathrm{hPa})$ is the pressure at the model top. The triply nested domains have the $(x, y)$ dimensions of $82 \times 64,124 \times 94$, and $124 \times 94$ with the grid sizes of 54, 18, and $6 \mathrm{~km}$, respectively. The model physics include (i) the Tao-Simpson cloud microphysics scheme (Tao and simpson, 1993) for the 6-km grid mesh, (ii) the Blackadar planetary boundary layer (PBL) parameterization (Zhang and Anthes 1982), and (iii) a cloud-radiation interaction scheme (Dudhia 1989). The sea-surface temperatures (SST) are held as constant in time during the 72-h integration. The model is initialized at 1200 UTC 21 August 1992 with a bogussed hurricane vortex. See Liu et al. (1997) for more details.

Hurricane Andrew, which landed on southern Florida on 24 August 1992, was the third most expensive hurricane to cross the United States coastline in the past 125 years. It produced a total of $\$ 25$ billion in property damage and took 15 lives. The storm originated from a tropical disturbance near the west coast of Africa on 14 August, and it was classified as Tropical Storm Andrew on 17 August. It reached hurricane strength on 22 August with its central pressure fall of $92 \mathrm{hPa}$ from 0000 UTC 21 to 1800 UTC 23 August. Andrew attained its maximum intensity of $922 \mathrm{hPa}$ with a maximum surface wind of $67 \mathrm{~m} \mathrm{~s}^{-1}$ prior to its landfall. Then, Andrew weakened by one category in the process of crossing Florida due to the strong surface friction and less surface sensible and latent heat fluxes over land, and strengthened again after moving into the Gulf of Mexico. For a more detailed description of the storm, please see Willoughby and Black (1996) and Liu et al. (1997).

\section{Experiment design}

In their simulation of Hurricane Andrew (1992), Liu et al (1997) used 23 vertical $\sigma$ layers. For the present study, we define a control run, in which 47 uneven $\sigma$ levels with higher resolution in the PBL are used (Exp. CTL46). The $47 \sigma$ levels are placed with 


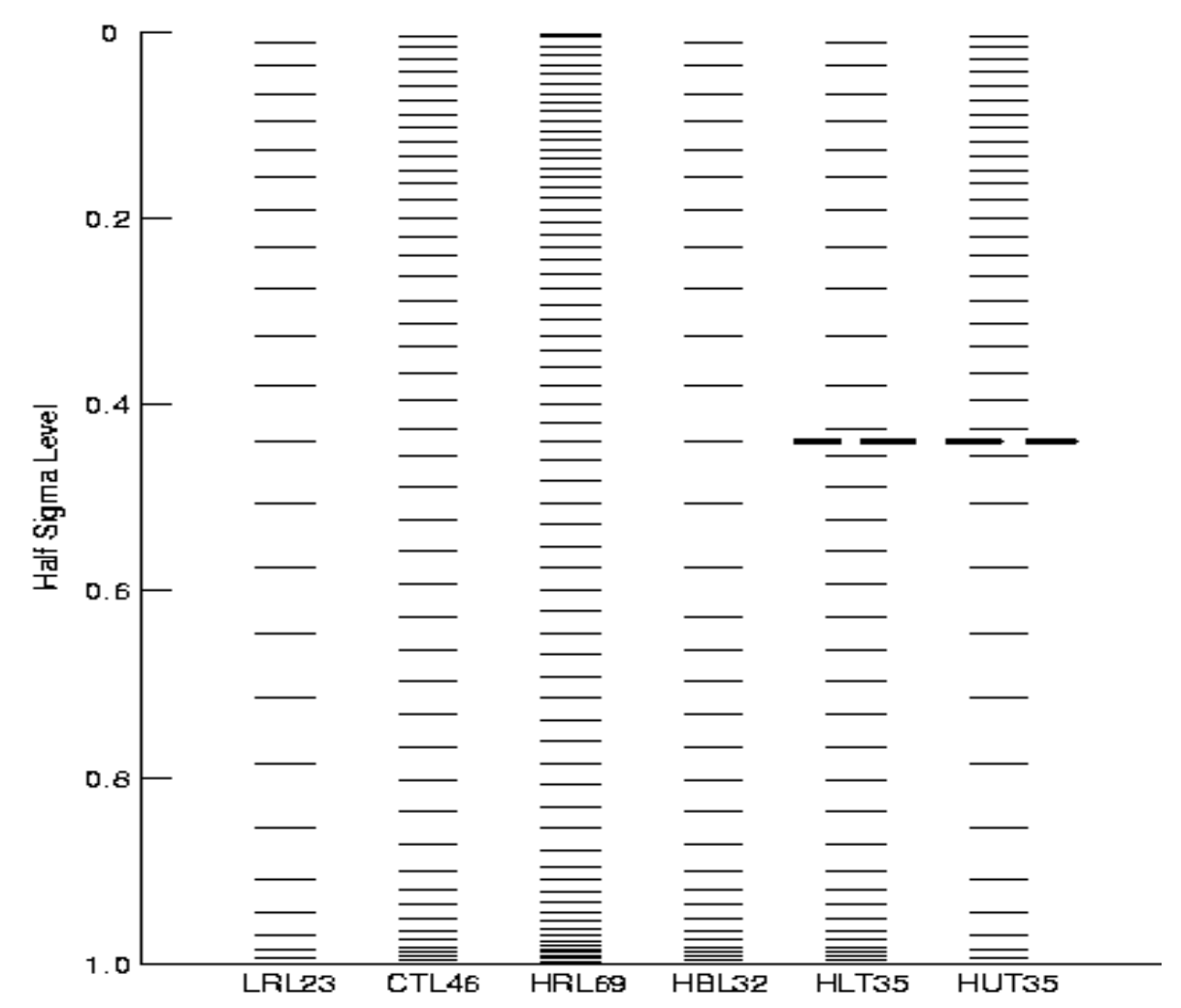

Fig. 1. Vertical distribution of half- $\sigma$ levels for each sensitivity experiment; see Table 1 for the experiment codes. Dashed lines denote roughly the location of the melting layer.

Table 1. Experimental design

\begin{tabular}{ll}
\hline Code & Description of experiment \\
\hline CTL46 & Control simulation with 46 vertical layers \\
HRL23 & The CTL46 resolution is evenly halved to 23 layers \\
HUT35 & The CTL46 resolution is evenly increased to 69 layers \\
HLT35 & Upper-level vertical resolution from LRL23 is doubled to a total of 35 layers \\
HBL29 & Lower-level vertical resolution from LRL23 is doubled to a total of 35 layers \\
DLT46 & The boundary-layer resolution from LRL23 is doubled to a total of 29 layers \\
\hline
\end{tabular}

the values of $1.0,0.995,0.99,0.985,0.98,0.97,0.96$, $0.945,0.93,0.91,0.89,0.855,0.82,0.785,0.75,0.715$, $0.68,0.645,0.61,0.575,0.54,0.505,0.47,0.44,0.41$, $0.38,0.35,0.325,0.3,0.275,0.25,0.23,0.21,0.19,0.17$, $0.155,0.14,0.125,0.11,0.095,0.08,0.065,0.05,0.035$, $0.02,0.01$, and 0 , which give 46 uneven half- $\sigma$ levels (see Fig. 1).

Several sensitivity experiments, as described in Table 1 and Fig. 1, are designed to study the effects of varying vertical resolutions on the simulated hurricane intensity and inner-core structures. In Exp. HRL69, 23 vertical layers are evenly added to CTL46, whereas in Exp. LRL23, the CTL46 vertical resolu- tion is halved evenly, i.e., Exp. LRL23 has the same resolution as that used in Liu et al. (1997). In addition, another three sensitivity experiments are conducted to examine the effects of varying vertical resolutions in different portions of the troposphere on the hurricane intensity and structures: (i) use the same vertical resolution as that in Exp. CTL46 above the melting level (roughly at $\sigma=0.44$ ) but keep the same resolution as that in Exp. LRL23 for the layers below (Exp. HUT35, i.e., higher resolution in the upper troposphere); (ii) use the same vertical resolution as that in Exp. CTL46 below the melting level but keep the same resolution as that in Exp. LRL23 for the lay- 
ers above (Exp. HLT35, i.e., higher resolution in the lower troposphere); and (iii) double the vertical resolution in the lowest $150 \mathrm{hPa}$ layer, i.e., up to $\sigma=0.845$ (Exp. HBL29). Figure 1 illustrates the distribution of the vertical $\sigma$-layers for all sensitivity simulations. In the above sensitivity simulations, all of the other model parameters are kept the same as those in Exp. CTL46.

It should be mentioned that based on the resolution consistency criterion (1), the vertical resolution for a grid size of $6 \mathrm{~km}$ should be about $60 \mathrm{~m}$. Clearly, the highest vertical resolution used herein (i.e., Exp. HRL69) is still much coarser than theoretically required, but it already pushes the existing computing power to the limit. Thus, our study will be limited to the highest vertical resolution possible with the current computing resources that are available to us.

A time step of 120 seconds is used to integrate the model for the outermost domain for all of the sensitivity simulations except for Exp. HRL69 in which a time step of 60 seconds is used in order to meet the computational stability criterion. The time step for the nested domains is always $1 / 3$ of that used for their mother domain. In view of the significant effect of using different time steps on the simulation of precipitation (Xu et al., 2001), one more sensitivity experiment is performed, in which the time step in Exp. CTL46 is reduced by half (Exp. DLT 46).

\section{Results}

In this section, we investigate the impact of varying vertical resolutions and time-step sizes on the simulation of Hurricane Andrew (1992) in terms of its intensity, eyewall structures, and heating profiles. The simulated tracks exhibit little sensitivity to the vertical resolution (with differences of 10-30 km among the simulations), except for Exp. HRL69 in which the storm is about $100 \mathrm{~km}$ slower and $80 \mathrm{~km}$ to the south of the control storm at the end of the 72-h simulation (see Wang 2002). The DLT46 storm also departs from the control by $80-100 \mathrm{~km}$ (not shown). In the following, maps of the time series and horizontal and vertical cross sections will be shown, mostly from the 54-h integrations at which time the storm is just about to experience the frictional influence of the Florida peninsula.

\subsection{Impact of vertical resolution}

Figure 2 compares the time series of the simulated minimum central pressures from the resolution sensitivity experiments. Several interesting sensitivity characteristics among the simulations are worthy of discussion. First, the simulated hurricane intensities depart more significantly with time between differ- ent experiments; the maximum intensities could range from the deepest $899 \mathrm{hPa}$ in Exp. HRL69, to 907 $\mathrm{hPa}$ in Exp. CTL46 and the weakest $932 \mathrm{hPa}$ in Exp. HUT35. The time series of central pressure from Exps. LRL23 and HRL69 are almost symmetrically distributed above and below that of Exp. CTL46, respectively. Of importance is that the deepest 899$\mathrm{hPa}$ central pressure reaches the minimum attainable sea-level pressure (or maximum potential intensity, Emanuel 1986) calculated from the prevailing SST. This indicates that some parameterized model physical processes (e.g., cloud microphysics or the PBL) may be too sensitive to the vertical resolution, suggesting the necessity to improve these parameterization schemes. Moreover, the central pressure difference from the two extreme simulations is as large as $33 \mathrm{hPa}$ in the first 60-h integration, and it retains its amplitude even several hours after landfall. This trend of departure would likely continue if the Florida peninsula were absent.

Second, increasing the vertical resolution in the low troposphere (HLT35) from LRL23 yields an intensity time series similar to that of HRL69, only a few $\mathrm{hPa}$ weaker in the first 60-h integration, implying the significant impact of changing the lower-level vertical resolution on the intensity prediction. Of interest is that increasing the upper-level vertical resolution (HUT35) from LRL23 even produces the weakest storm, i.e., $14 \mathrm{hPa}$ weaker than that in LRL23, despite the use of more vertical layers. Of further interest is that the HUT35 time series follows closely that of LRL23 during the first 30-h integration, deepens slightly from 30-36 h, but becomes 10-20 hPa weaker than the LRL23 storm afterward. An examination of the model-simulated radar reflectivity maps reveals that this bifurcation is caused by different cloud structures in the eyewall and spiral rainbands as a result of different vertical resolutions. For example, the model generates a partial eyewall in HUT35, but a near-full eyewall in Exp. LRL23 with marked differences in size and rainband distribution from the 39-h integration, which is just a couple of hours before the crossover of the sea-level pressure time series (cf. Figs. 2 and 3). Similarly, the time series in Exps. HLT35 and HBL29 are similar to that of CTL46 in the first $36 \mathrm{~h}$, but then both become significantly deeper. Despite the use of less vertical layers in HLT35 and HBL29, their final intensities are close to the intensity in HRL69. The results suggest that (i) increasing the vertical resolution in the lower troposphere is more efficient than that in the upper levels in deepening a hurricane, and (ii) different partitionings of a given number of vertical layers could have different impacts on the deepening rates and cloud structures in the eyewall and rainbands during the different stages of hurricane development. 


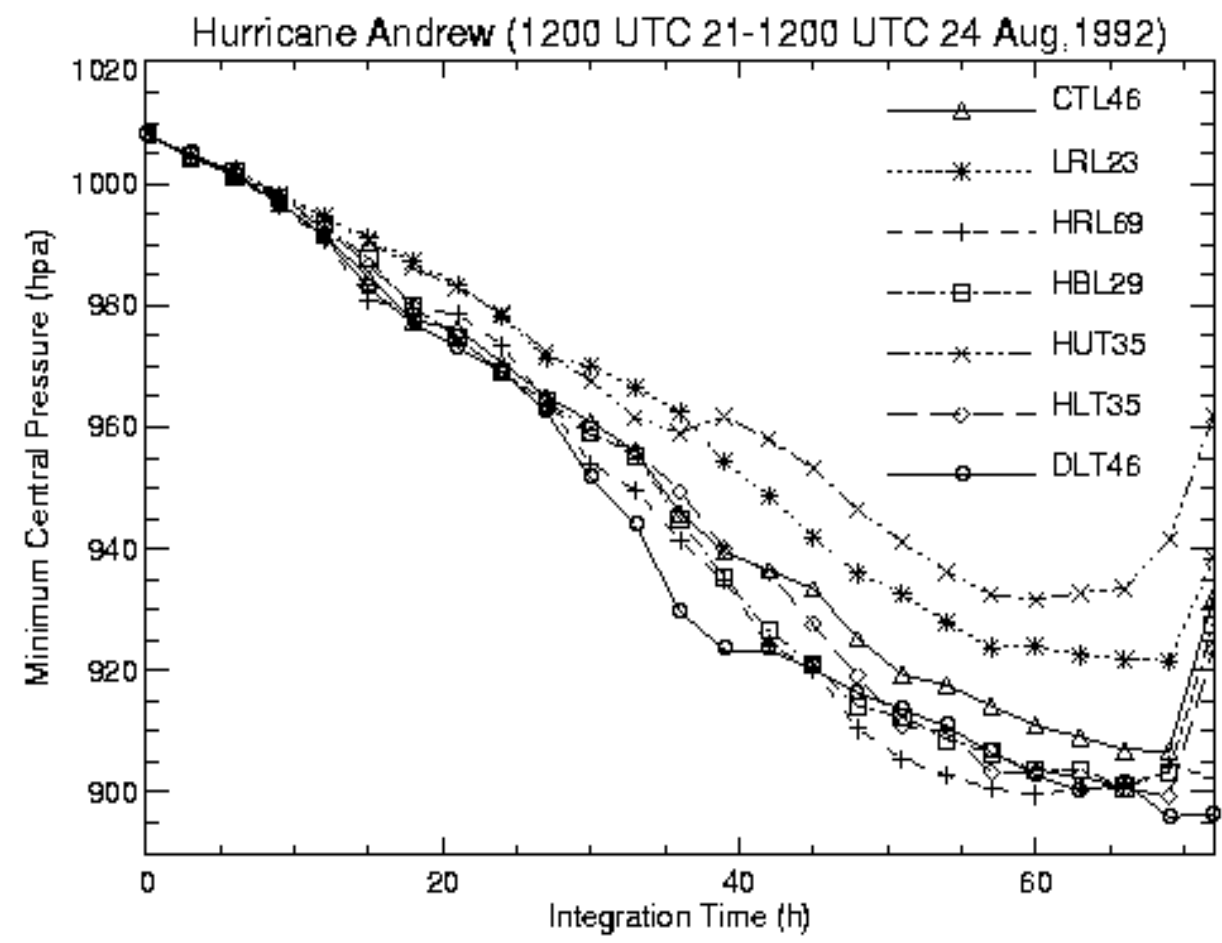

Fig. 2. Three-hourly time series $(72-\mathrm{h})$ of the minimum surface pressure from all sensitivity experiments; see Table 1 for their codes.
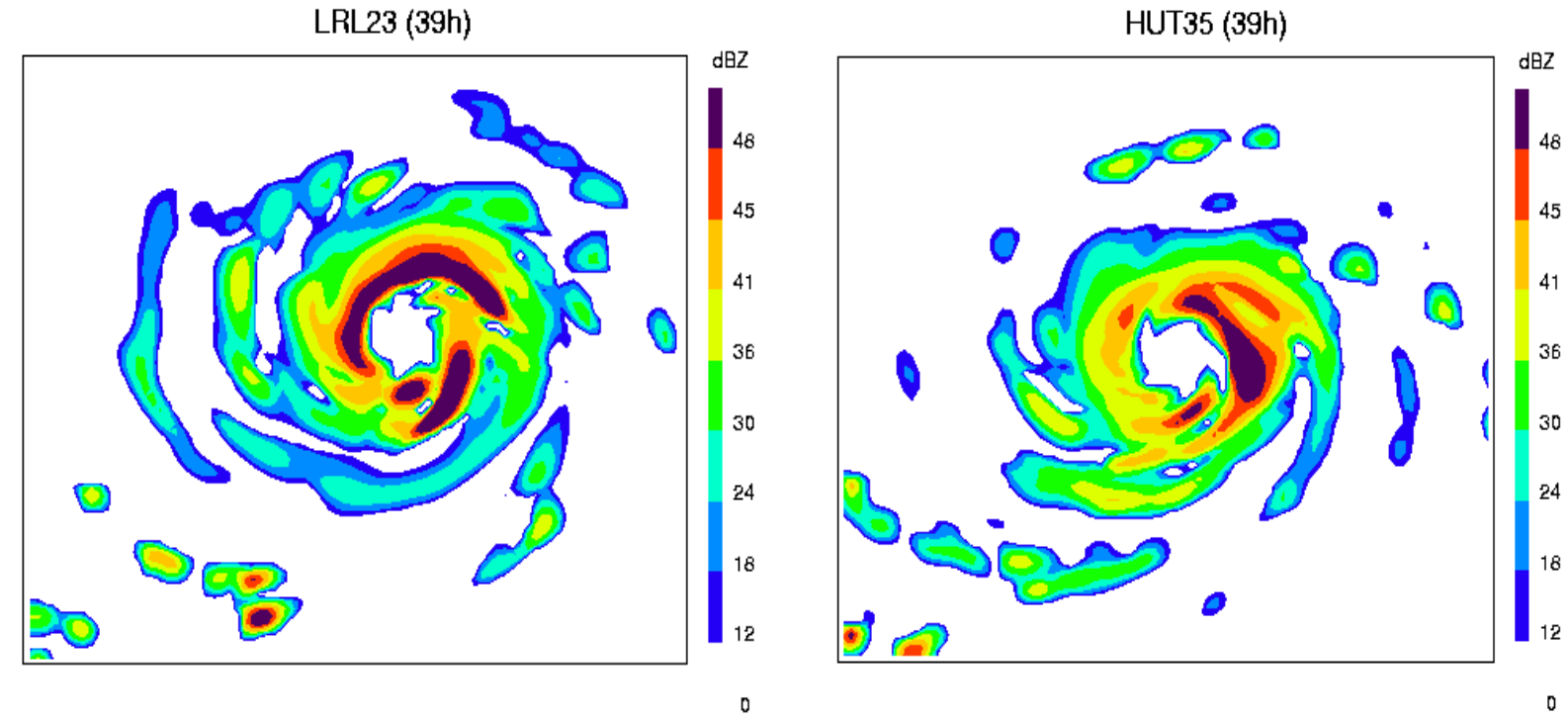

Fig. 3. Horizontal distribution of radar reflectivity, taken at $\sigma=0.785$ (i.e., near $800 \mathrm{hPa}$ ), from the 39 -h integration of (a) Exp. LRL23; and (b) Exp. HUT35.

The time series of simulated maximum surface winds, given in Fig. 4, shows the relation of the simulated hurricane intensity to the surface layer resolution and frictional effects. The use of the thickest surface layer (80 $\mathrm{m}$ at a full- $\sigma$ level) in Exp. LRL23 produces the greatest maximum surface wind of $75 \mathrm{~m} \mathrm{~s}^{-1}$ prior to landfall, whereas the thinnest surface layer (about $27 \mathrm{~m}$ ) in HRL69 has the weakest maximum surface wind of $63 \mathrm{~m} \mathrm{~s}^{-1}$ in spite of its deepest minimum pressure (cf. Figs. 2 and 4). This is consistent with the notion that the frictional effects would be more (less) pronounced if a thinner (thicker) surface layer of air 


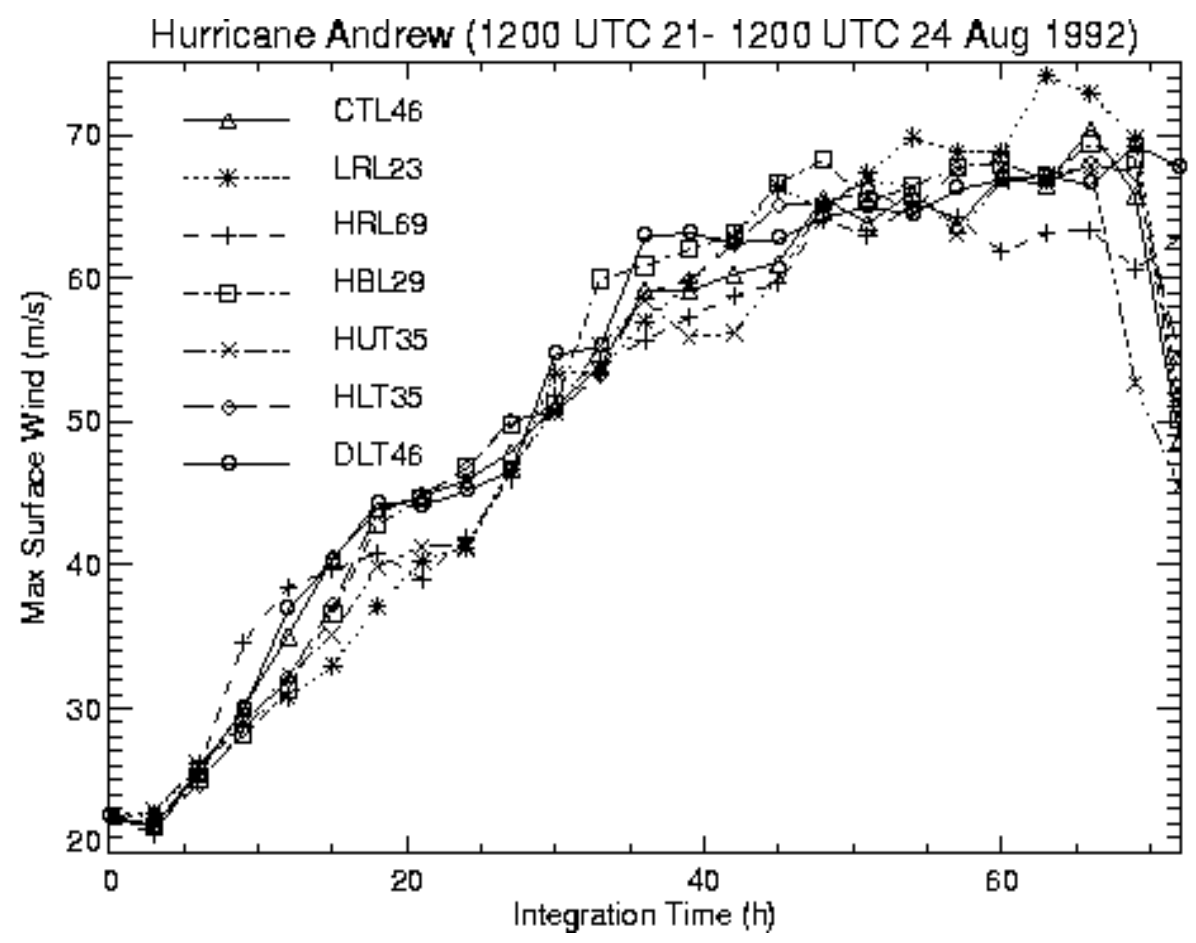

Fig. 4. As in Fig. 2, but for the maximum surface winds.

mass interacts with the bottom surface. In this regard, we acknowledge that it is more appropriate to use a thin (ideally $20 \mathrm{~m}$ ) surface layer instead of that used in Liu et al. (1997) when verifying the modeled surface winds against the observed at the altimeter level (i.e., $z=10 \mathrm{~m}$ ). On the other hand, since the surface winds are layer-averaged, the well-known surface logarithmic law could not be used to adjust them to an altimeter level as suggested by Powell and Houston (1999). For example, the maximum surface winds in HRL69 and LRL23 are not distinct from each other until after the 45 -h integration. Only at $63 \mathrm{~h}$, one may obtain similar maximum surface winds between HRL69 and LRL23 after they are converted to the altimeter level using surface similarity theory (see Powell and Houston, 1999). At other times, such an adjustment tends to underestimate substantially the magnitude of the simulated surface winds. Perhaps a better way to examine this issue should be to increase only the surfacelayer resolution from Exp. LRL23 such that differences in central pressures between the control and sensitivity simulations are small.

The simulated radar reflectivity from the 54-h integrations shows different inner-core structures of clouds and precipitation among the various experiments (see Fig. 5). It is evident that the eyewall convection becomes more intense, more compact, and more symmetric with a wider annulus of clouds outside as the vertical resolution increases from 23 to 69 layers. Different inner-core cloud/precipitation structures also appear in the other three sensitivity runs. For example, the eyewall convection in HBL29 is also near-symmetric with more convection occurring to the west, whereas there is a tendency to develop a partial double eyewall to the east in HLT35 (not shown), as also hinted from Fig. 2. Surface rainfall amounts and distribution, including major spiral rainbands, also differ between the simulations (not shown). These results are all consistent with the simulated intensity changes, as expected.

Figures 6-8 display the height-radius cross sections of tangential, radial, and vertical winds, respectively, superposed with the in-plane flow vectors from the 54-h experimental integrations. Results from Exp. HLT35 are not shown because its cross-sectional features and amplitudes are similar to those in HBL29. These maps exhibit a typical hurricane structure: an intense radial inflow in the PBL, an outflow jet near the top of the PBL where the tangential winds are peaked, a slantwise updraft with a negative shear in horizontal winds in the eyewall, and an outflow layer in the upper troposphere (see Liu et al., 1999). In general, the sensitivity of the simulated flow intensity to vertical resolution is consistent with that of the minimum central pressure. For instance, the peak tangential wind increases from $60 \mathrm{~m} \mathrm{~s}^{-1}$ in LRL23 to 80 $\mathrm{m} \mathrm{s}^{-1}$ in CTL46, and $90 \mathrm{~m} \mathrm{~s}^{-1}$ in HRL69 (Fig. 6), whereas the peak PBL inflow increases from $30 \mathrm{~m} \mathrm{~s}^{-1}$ 

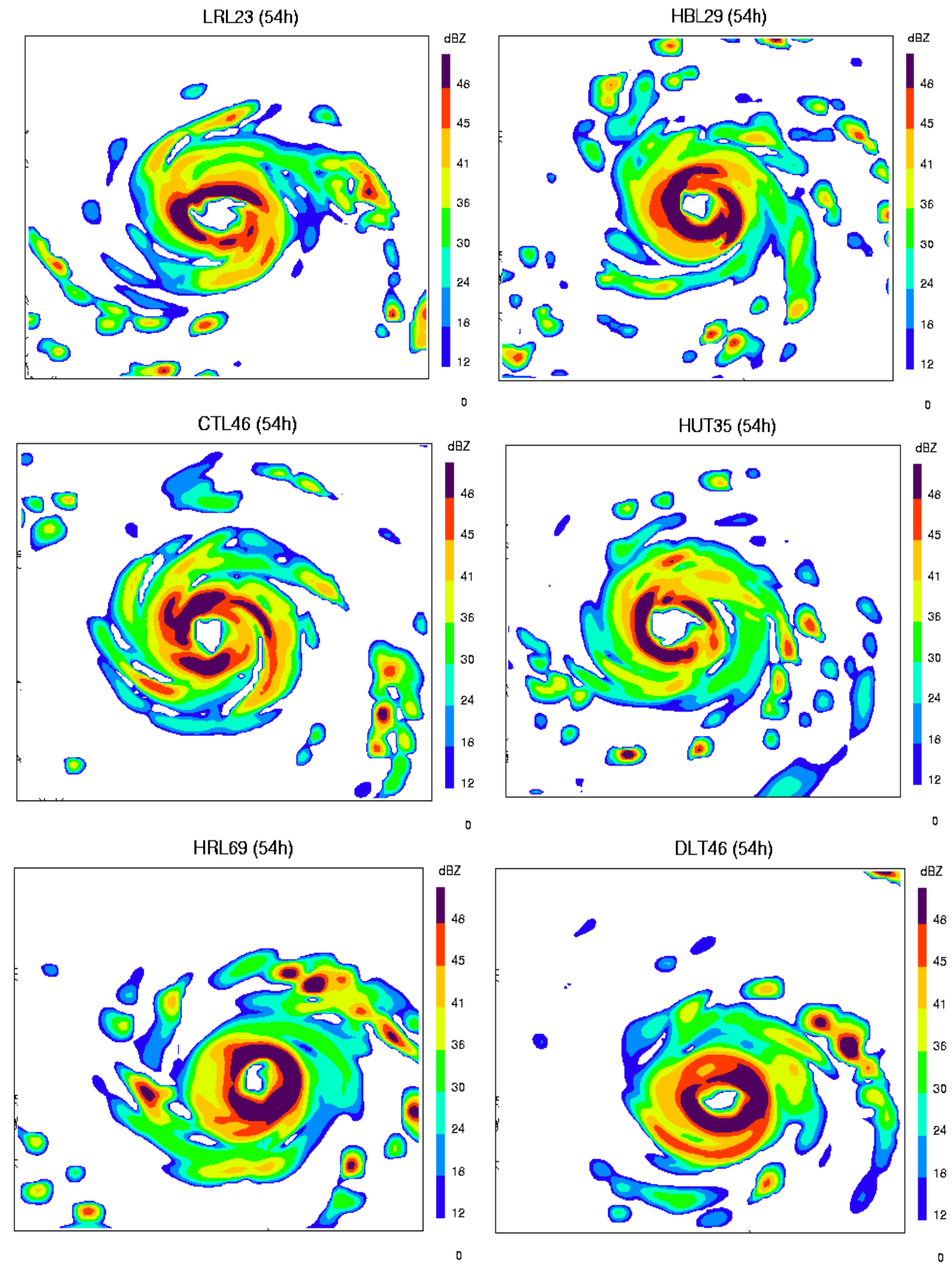

Fig. 5. Horizontal distribution of radar reflectivity, taken at $\sigma=0.785$ (i.e., near $800 \mathrm{hPa}$ ), from the 54 -h integrations of all sensitivity experiments except for Exp. HLT35. 

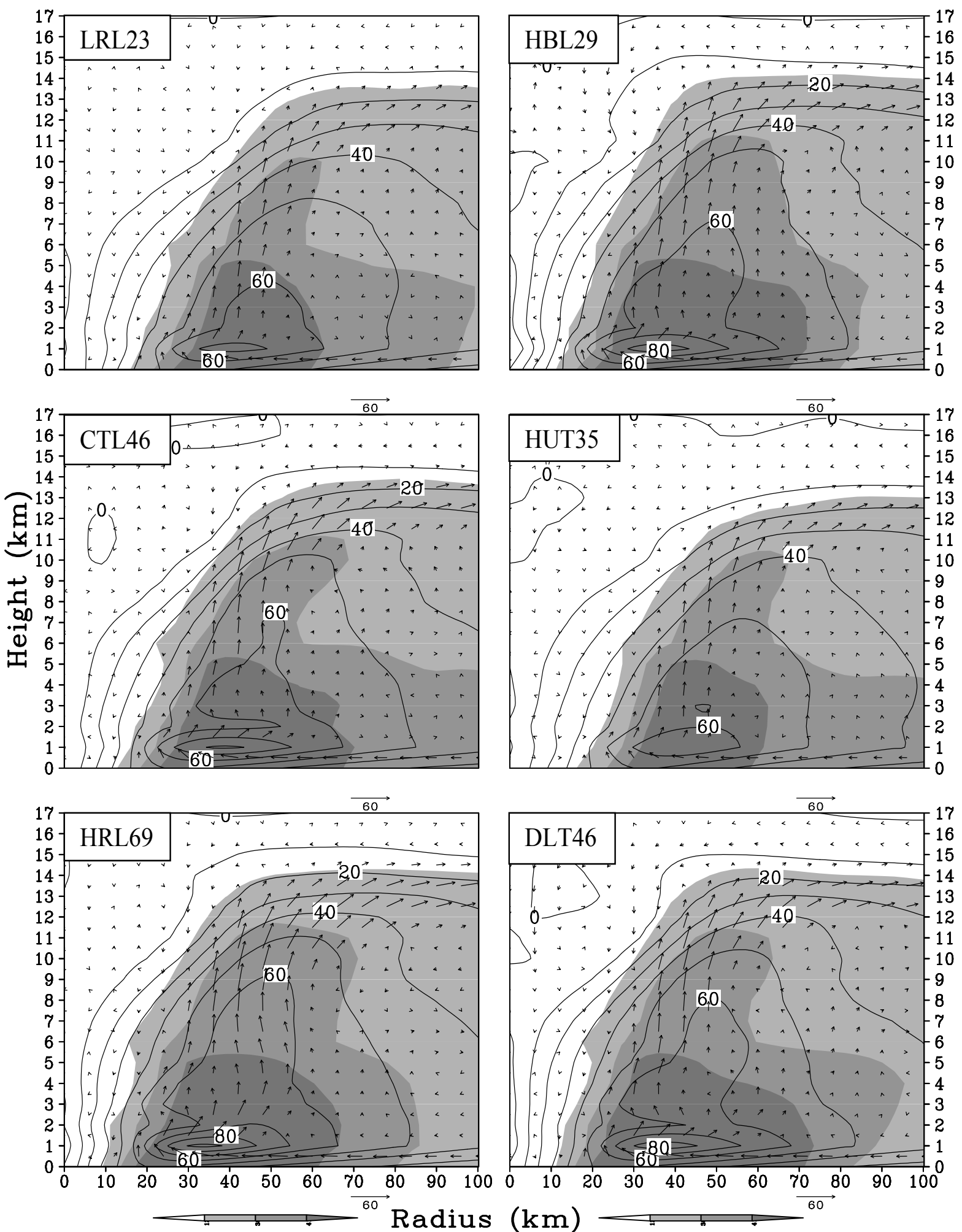

Fig. 6. Height-radius cross sections of tangential winds, at intervals of $10 \mathrm{~m} \mathrm{~s}^{-1}$, superposed with the in-plane flowvectors, from the 54-h integrations of all sensitivity experiments except for Exp. HLT35. 

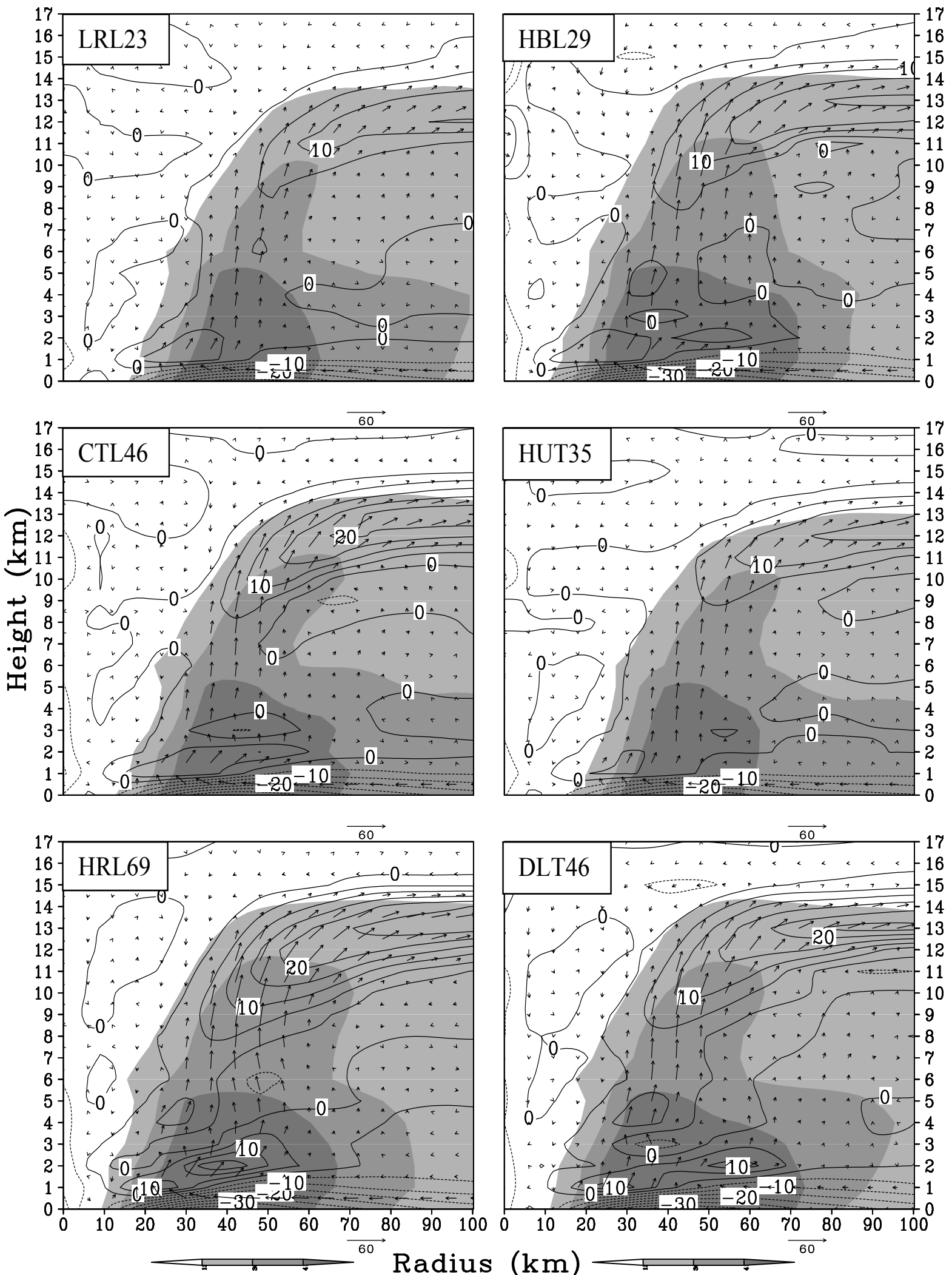

Fig. 7. As in Fig. 6, but for radial winds at intervals of $5 \mathrm{~m} \mathrm{~s}^{-1}$. 

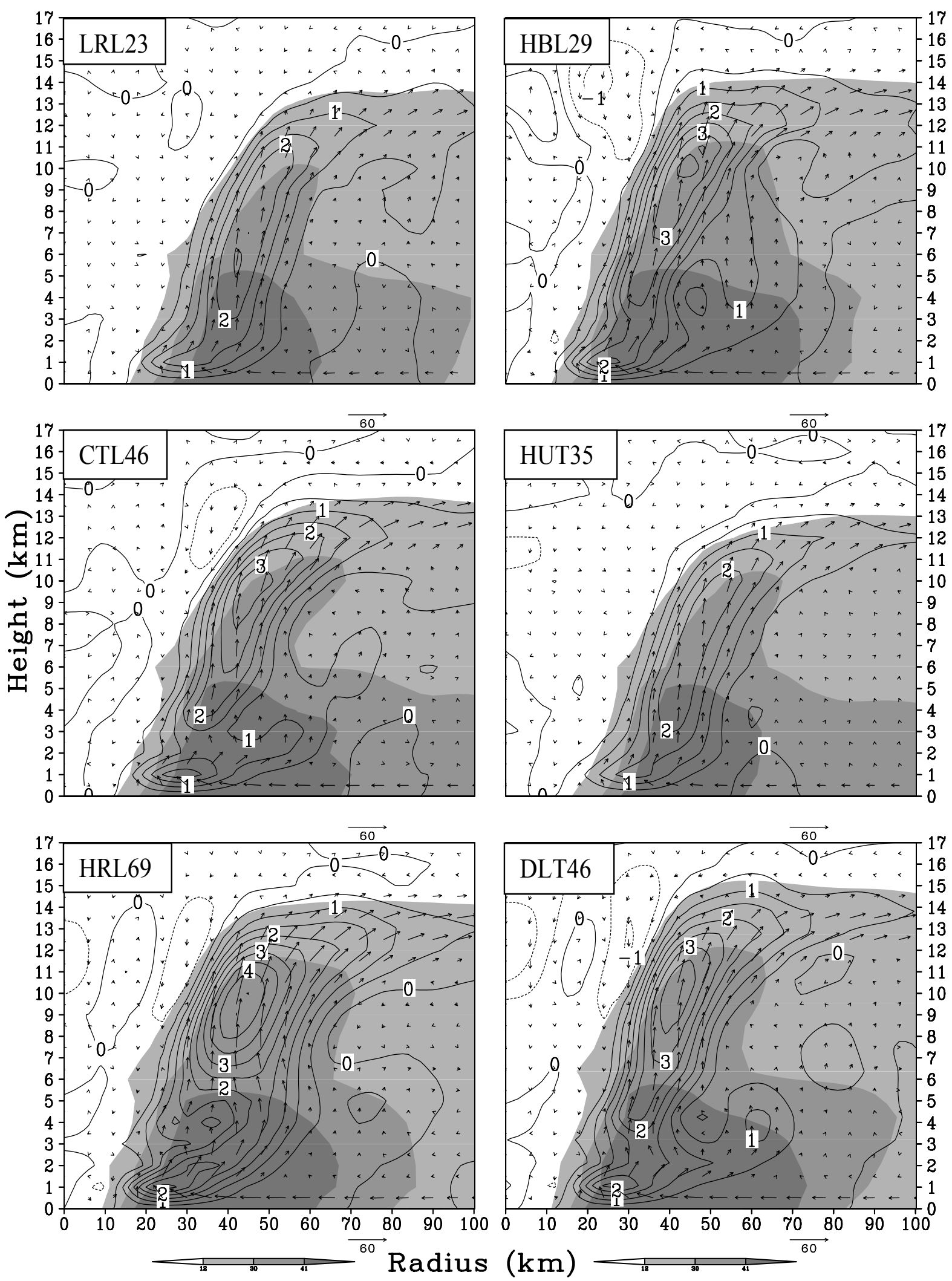

Fig. 8. As in Fig. 6, but for vertical velocity at intervals of $0.5 \mathrm{~m} \mathrm{~s}^{-1}$. 


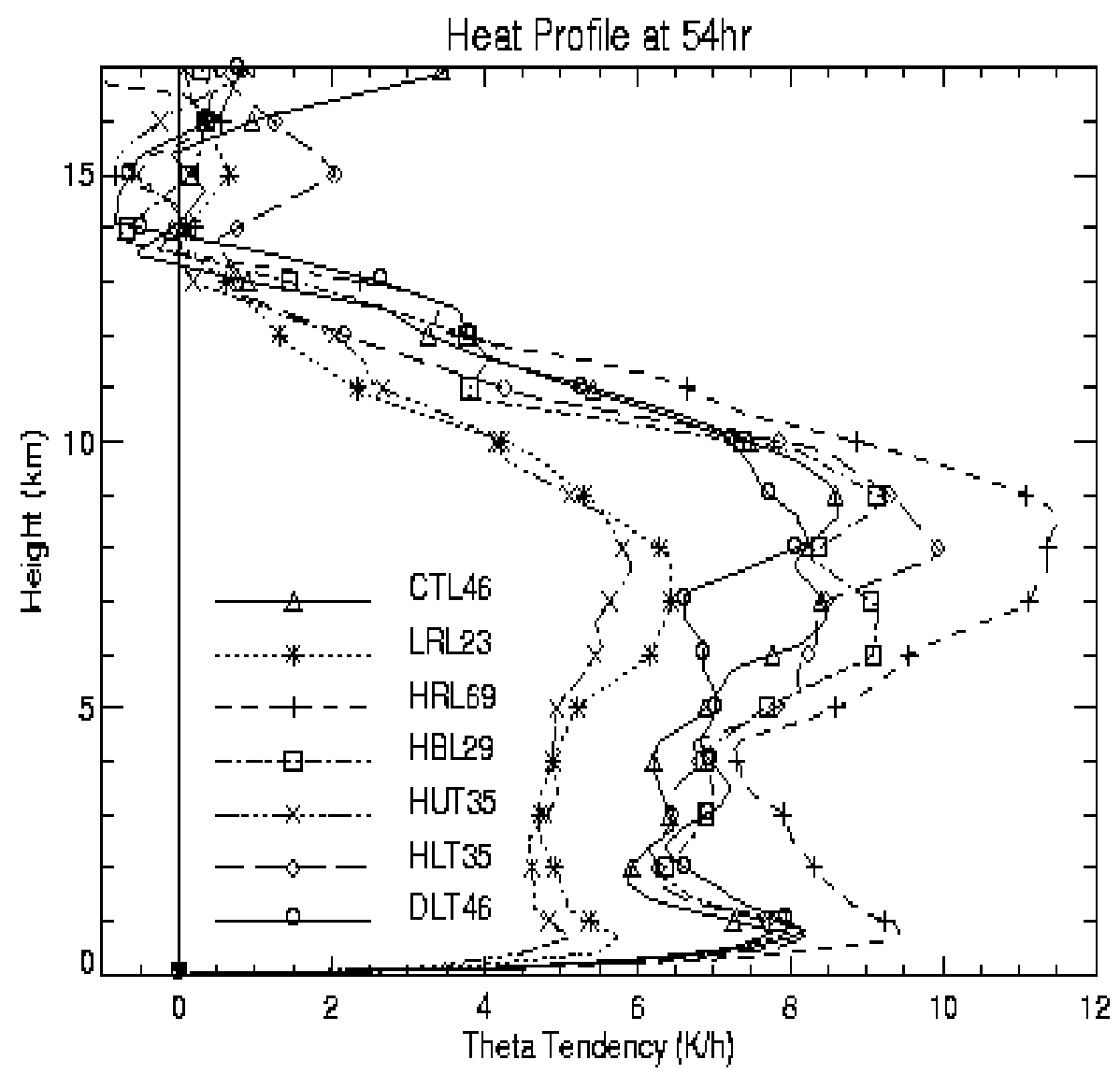

Fig. 9. Vertical profiles of the latent heating rates $\left(\mathrm{K} \mathrm{h}^{-1}\right)$ averaged within a radius of $150 \mathrm{~km}$ from the hurricane centers from the 54-h integrations of all sensitivity experiments.

in LRL23 to $40 \mathrm{~m} \mathrm{~s}^{-1}$ in HRL69 (Fig. 7). Similarly, the low-level outflow jet and the upper-level outflow increase substantially, both nearly double as the vertical resolution increases from 23 to 69 layers. The eye sizes are smaller for more intense storms (Fig. 8).

Increasing the upper-level resolution (HUT35) has less notable impact on the height-radius distribution of horizontal winds and vertical motion, as compared to LRL23 (cf. Figs. 6-8). On the other hand, increasing the low-level resolution (i.e., HBL29 or HLT35) generates the amplitudes of the low-level horizontal wind that are similar to those in CTL46, as expected. However, despite the development of a more intense storm in HBL29 (and HLT35), its associated upper-level outflows are similar to those in LRL23 but markedly weaker than those in CTL46. This indicates the importance of designing a comparable distribution of vertical resolution in studying hurricanes' inner-core structures. Better results tend to be obtained when high-resolution layers are used throughout the troposphere.

When properly designed, increasing vertical reso- lution tends to increase the magnitude of vertical motion, for example, the maximum updraft varies from $2.5 \mathrm{~m} \mathrm{~s}^{-1}$ in LRL23 to $4.5 \mathrm{~m} \mathrm{~s}^{-1}$ in HRL69 (Fig. 8). Doubling the low-level resolution (HBL29 and HLT35) can also duplicate somewhat the eyewall structure and intensity shown in higher resolution runs (e.g., CTL46). Again, doubling the upper-level resolution (HUT35) from LRL23 affects little the vertical structure and amplitude of vertical motion in the eyewall. Of interest is that a well-defined secondary peak updraft, corresponding to the peak tangential wind and an outflow jet, occurs near the top of the PBL in all high-resolution runs except in HUT35. This peak updraft does not seem to be related to any computational instability, because this feature still appears in Exp. DLT46 in which the time step of 13.3 seconds for the finest $6-\mathrm{km}$ resolution domain is reduced by half (i.e., 6.7 seconds) (cf. CTL46 and DLT46 in Fig. 8). (Note though that the same time step of 6.7 seconds is also used for HRL69.) This feature results more likely from the Ekman pumping processes, which is associated with the intense cyclonic vorticity generated by 
the peak tangential winds and a radial outflow jet near the top of the PBL (see Zhang et al., 2001), that are then enhanced by diabatic heating.

Figure 9 shows the storm-scale averaged heating profiles from each sensitivity run which represent the collective effects of deep convection on the large-scale environment. These profiles exhibit a deep layer of intense latent heating up to an altitude of $13 \mathrm{~km}$ in the eyewall, with a bimodal heating distribution: one associated with the low-level outflow jet and the other in the upper-level outflow layer. All these are similar to those shown in Zhang et al. (2002) except for their magnitudes due to the use of different radii for the area averages. Evidently, the heating rates depend highly on the vertical resolution in the same way as the storm intensity. Namely, the higher the vertical resolution, the more intense the latent heating and a stronger storm results, since hurricanes are driven by latent heat release. The heating profiles from the other runs, particularly HRL69, are systematically much greater in magnitude than those from LRL23 and HUT35 throughout the troposphere. Again, the vertical heating profiles from Exps. LRL23 and HUT35 are almost identical in structure and magnitude; similarly among CTL46, HLT35, and HBL29 up to $z=7 \mathrm{~km}$, as could be expected from their similar resolutions in the low troposphere.

While increasing the upper-level resolution has little impact on the eyewall structure, it does increase slightly the heating rates in the upper outflow layer (e.g., HUT35 vs. LRL23, CTL46 vs. HLT35 and HBL29). This result appears to suggest that the use of higher vertical resolution helps trigger the grid-box depositional growth (condensation) and sublimation (evaporation). For example, consider two extreme cases for a given relative humidity: a very thick and a very thin layer. The grid-box saturation will likely occur first in the thin layer if all the other conditions are identical. Of course, the decrease in truncation errors leading to the generation of stronger divergence, as the vertical resolution increases, may also contribute positively to the magnitude of latent heating and the storm intensity. Nevertheless, it is well known that the low-level heating maximum is more efficient than the upper-level one in spinning up mesoscale cyclones (Tracton 1973; Anthes and Keyser 1979; Zhang and Fritsch, 1988a). The more intense low-level heating with the increased local resolution is consistent with the increased hurricane intensity shown in Figs. 2 and 8.

Since the hurricane eyewall may be viewed as an atmospheric "front", it would be desirable to see if the resolution consistency, discussed by the previous studies mentioned in Section 1, could apply to the simulated hurricane. For this purpose, Fig. 10 shows vertical cross sections of potential temperature deviations from two extreme resolution simulations but with similar intensities, namely, HRL69 and HBL29. It is evident that more significant wavy structures in isotherms, horizontal thermal gradients, and in-plane flow vectors develop in the upper troposphere in Exp. HBL29, and they are indicative of inertial gravity waves. These wavy structures are, to a certain degree, consistent with those found by Persson and Warner (1991). In contrast, the low- to mid-level features in the two storms, including the warm cores in the eye and thermal gradients across the eyewall, are smoothly distributed and similar to each other in magnitude and structure. The absence of the wavy structures below the peak updraft level appears to be attributable to the facts that the swirling winds in the eyewall are inertially stable and that any disturbance, once developed, could be quickly smoothed out by the swirling winds through the axisymmetrization process (Smith and Montgomery, 1995). They appear to be the two major differences between highly and non- or weakly rotating weather systems.

\subsection{Impact of time step size}

$\mathrm{Xu}$ et al. (2001) found that numerical simulation of an MCS, occurring in a weakly forced large-scale environment, is sensitive to the time-step size even within the computational stability constraint. Of particular interest is that as the time step decreases, the movement, longevity, and precipitation of their simulated MCS depart further from the observations and the control-simulated MCS. Thus, we are motivated to conduct Exp. DLT46 in which the time-step size is halved from Exp. CTL46. As mentioned earlier, reducing the time step slows the movement of the storm, which is consistent with the case in Xu et al. (2001). They attributed this sensitivity to the time step dependent formulation of the numerical diffusivity. However, this attribution cannot explain the development of the DLT46 storm that is $15 \mathrm{hPa}$ (at $39 \mathrm{~h}$ ) and 10 hPa (after $48 \mathrm{~h}$ ) deeper than that in CTL46 (see Fig. 2 ), although the differences in their maximum surface winds are small (about $2-5 \mathrm{~m} \mathrm{~s}^{-1}$ ). This is because the increased numerical diffusivity tends to smooth out meteorological fields or weaken the storm, as also indicated by the results of $\mathrm{Xu}$ et al. (2001). Note that the DLT46's final intensity is much stronger than that in CTL46 due to its southwestward-shifted track without experiencing landfall; similarly for the HRL69 storm (see Figs. 2 and 4). Thus, the DLT46's final minimum surface pressure is close to that of the HRL69 storm. 


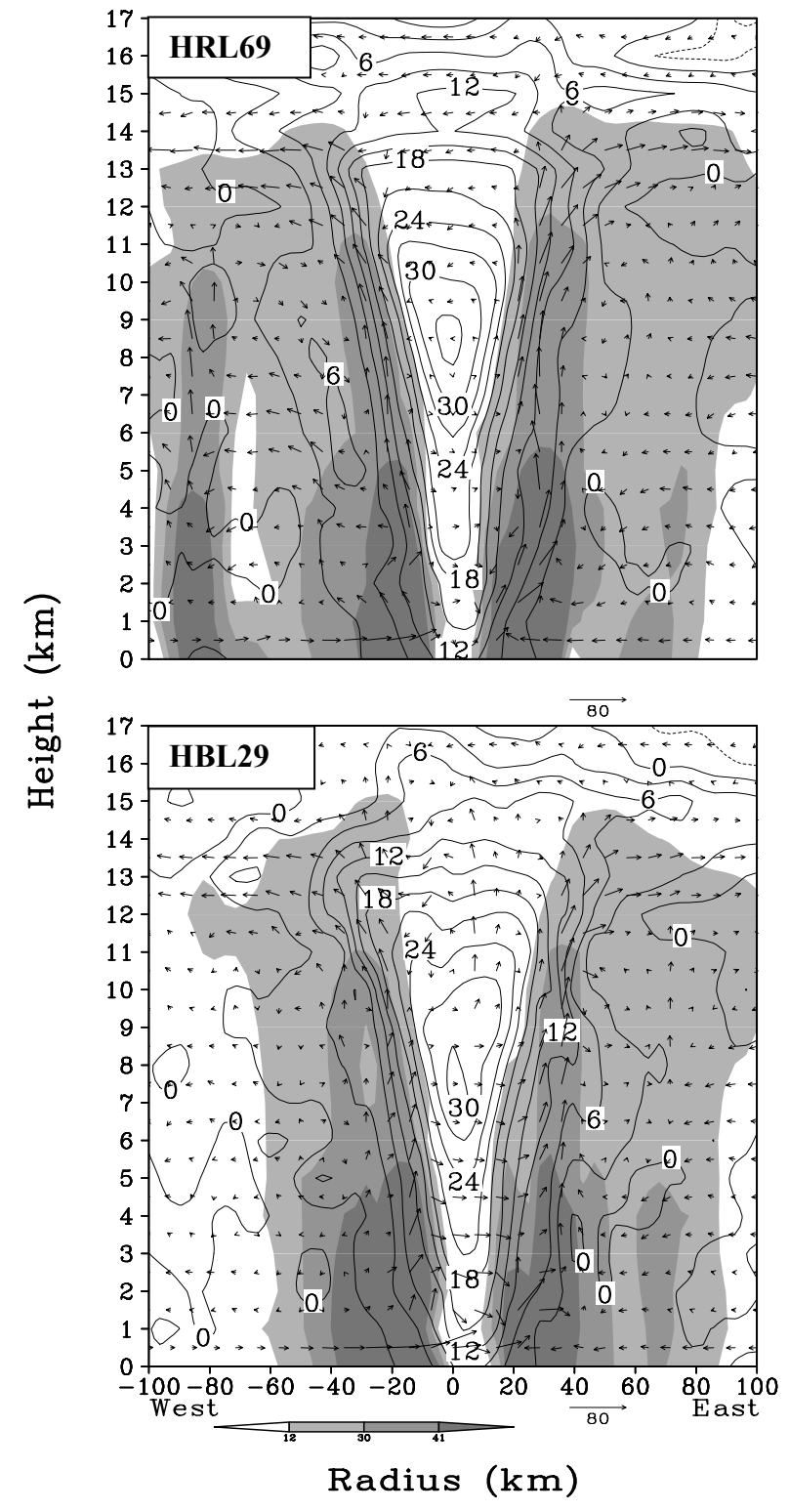

Fig. 10. West-east cross sections of potential temperature $(\theta)$ deviations, at intervals of $3 \mathrm{~K}$, superposed with in-plane flow vectors from the 54-h integrations of Exps. HRL69 and HBL29.

Corresponding to its deeper central pressure, DLT46 exhibits more organized clouds and precipitation in the eyewall (Fig. 5), and stronger horizontal and vertical motion (Figs. 6-8) than those in CTL46. Similarly, the vertical heating profile in DLT46 shows more latent heat release below $z=5 \mathrm{~km}$ and less above compared to that in CTL46. This suggests that reducing the time-step size would facilitate grid-box saturation, which may be achieved by (a) the improved truncation errors, and (b) the more accurate calculation of phase changes. In principle, phase changes should be iteratively calculated between latent heating increments and phase-change amounts. However, they are calculated in the Tao-Simpson microphysics scheme by a simple saturation adjustment scheme (Tao et al., 1989). The result suggests the necessity of improving the calculations of phase changes, perhaps more importantly in the lower troposphere because of its more significant roles in deepening cyclones than in the upper troposphere.

\section{Summary and conclusions}

In this study, several 72-h numerical integrations are performed to study the sensitivity of the simulated Hurricane Andrew (1992) to various vertical resolutions and time-step sizes using the nested grid, cloud resolving version of the PSU/NCAR nonhydrostatic model (i.e., MM5) with the finest grid size of $6 \mathrm{~km}$. The vertical resolution varies from 23 to 69 layers, with varying layer thicknesses in the lower and upper portions of the troposphere, whereas the time-step size is halved in two sensitivity tests.

Detailed analyses of the sensitivity experiments show that changing vertical resolution has little impact on the hurricane track except for the two most intense storms. However, the hurricane intensity and inner-core cloud/precipitation structures are very sensitive to the vertical resolution. Specifically, increasing vertical resolution tends to simulate a deeper storm in terms of central pressure, and three-dimensional winds with more precipitation. For the vertical resolutions tested herein, the surface central pressure ranges from 932 to $899 \mathrm{hPa}$, the azimuthally averaged peak values of the tangential wind from 60 to $90 \mathrm{~m} \mathrm{~s}^{-1}$, the PBL inflow from 30 to $40 \mathrm{~m} \mathrm{~s}^{-1}$, the updraft from 2.5 to $4.5 \mathrm{~m} \mathrm{~s}^{-1}$, and the diabatic heating rates from 50 to $80 \mathrm{~K} \mathrm{~h}^{-1}$. Similarly, the low-level outflow jet and the upper-level outflow increase significantly, both nearly double as the vertical resolution increases from 23 to 69 layers. Of importance is that the deepest storm simulated reaches the maximum potential intensity calculated from the prevailing sea-surface temperature, and this trend would continue as the vertical resolution further increases, indicating that some parameterized model physical processes (e.g., cloud microphysics or the PBL) may be too sensitive to the vertical resolution.

It is found that increasing the vertical resolution in the low troposphere is more efficient in intensifying a hurricane, whereas changing the upper-level vertical resolution has little impact on the intensity prediction. The former case could cause more deepening of hurricanes because the low-level latent heating tends to induce more moisture convergence in the PBL where the latent energy source originates. On the other hand, 
the increased latent heating in the upper levels would cause the convergence of the mid-level cold and dry air, suppressing deep convection in the eyewall. With higher resolutions in the low troposphere, the model produces a wider eyewall, stronger spiral rainbands, and a wider area of precipitation as a result of a more intense low-level outflow jet being generated. It is shown that the noisy flows resulting from inconsistent resolutions found in the previous studies are only notable in the upper-level outflow where it is inertially less stable.

It is shown that the use of a thicker surface layer tends to produce the higher maximum surface wind; different surface layer thicknesses could produce the maximum winds ranging from $75 \mathrm{~m} \mathrm{~s}^{-1}$ (with $80 \mathrm{~m}$ ) to $60 \mathrm{~m} \mathrm{~s}^{-1}$ (with $27 \mathrm{~m}$ ). This is consistent with the notion that the frictional effects would be more (less) pronounced if a thinner (thicker) surface layer of air mass interacts with the bottom surface. This suggests that a thin surface layer be used, if possible, to verify against observations at an altimeter level $(z=10 \mathrm{~m})$.

It is also shown that the model-simulated hurricane intensity and inner-core structures are sensitive to the size of time step. Reducing the time-step size tends to increase the numerical diffusion due to the formulation used, and appears to facilitate grid-box saturation. This result suggests that any use of large time-step sizes, e.g., in the semi-implicit time integration or semi-Lagrangian numerical schemes, may cause large errors in the calculation of phase changes in the current cloud physics schemes, unless some iterations of phase changes are performed.

In conclusion, it is highly desirable to use higher vertical resolution and smaller time-step sizes when it is possible, together with higher horizontal grid resolution, to model tropical storms and the other MCSs more realistically. It should be mentioned, though, that the above conclusion may not be applicable in certain cases in which most of the precipitation is generated by convective parameterization in coarseresolution models.

Acknowledgments. This work was supported by the NSF grant ATM-9802391, the NASA grant NAG57842, and the ONR grant N00014-96-1-0746.

\section{REFERENCES}

Anthes, R. A., and D. Keyser, 1979: Tests of a fine-mesh model over Europe and the United States. Mon Wea. Rev., 107, 963-984.

Chou, M.-D., 1975: A study of the effects of vertical resolution and measurement errors on an iteratively inverted temperature profile. J. Atmos. Sci., 32, 419426.

Dudhia, J., 1989: Numerical study of convection observed during the winter monsoon experiment using a mesoscale two-dimensional model., J. Atmos. Sci., 46, 3077-3107.

Dudhia, J., 1993: A nonhydrostatic version of the Penn State-NCAR mesoscale model: Validation tests and simulation of an Atlantic cyclone and cold front. Mon. Wea. Rev., 121, 1493-1513.

Emanuel, K. A., 1986: An air-sea interaction theory for tropical cyclones. Part I: Steady-state maintenance. J. Atmos. Sci., 43, 585-604.

Hamilton, K., R. J. Wilson, and R. S. Hemler, 1999: Middle atmosphere simulated with high vertical and horizontal resolution versions of a GCM: Improvements in the cold pole bias and generation of a QBO-like oscillation in the tropics. J. Atmos. Sci., 56, 3829-3846.

Lane, D. E., R. C. J. Somerville, and S. F. Iacobellis, 2000: Sensitivity of cloud and radiation parameterizations to changes in vertical resolution. J. Climate, 13, 915922.

Lindzen, R. S., and M. S. Fox-Rabinovitz, 1989: Consistent vertical and horizontal resolution. Mon. Wea. Rev., 117, 2575-2583.

Liu, Y., D.-L. Zhang, and M. K. Yau, 1997: A multiscale numerical study of Hurricane Andrew (1992). Part I: An explicit simulation. Mon. Wea. Rev., 125, 30733093.

Liu, Y., D.-L. Zhang, and M. K. Yau, 1999: A multiscale numerical study of Hurricane Andrew (1992). Part II: Kinematics and inner-core structures. Mon. Wea. Rev., 127, 2597-2616.

Pecnick, M. J., and D. Keyser, 1989: The effect of spatial resolution on the simulation of uppertropospheric frontogenesis using a sigma-coordinate primitive equation model. Meteor. Atmos. Phys., 40, 137-149.

Persson, P. O. G., and T. T. Warner, 1991:Model generation of spurious gravity waves due to inconsistency of the vertical and horizontal resolution. Mon. Wea. Rev., 119, 917-935.

Powell, M. D., and S. H. Houston, 1999: Comments on "A multiscale numerical study of Hurricane Andrew (1992). Part I: Explicit simulation and verification" Mon. Wea. Rev., 127, 1706-1710.

Smith, G. B., II, and M. T. Montgomery, 1995: Vortex axisymmetrization and its dependence on azimuthal wavenumber or asymmetric radial structure changes. Quart. J. Roy. Meteor. Soc., 121, 1615-1650.

Tao, W.-K., and J. Simpson, 1993: The Goddard cumulus ensemble model. Part I: Model description. Terr. Atmos. Oceanic Sci., 4, 35-72.

Tao, W.-K., J. Simpson, and M. McCumber, 1989: An icewater saturation adjustment. Mon. Wea. Rev., 117, 231-235.

Tracton, M. S., 1973: The role of cumulus convection in the development of extratropical cyclones. Mon. Wea. Rev., 107, 572-593.

Wang, X., 2002: Sensitivity of numerical simulation of Hurricane Andrew (1992) to varying vertical resolution. M.S. Scholarly Paper, University of Maryland, 49pp.

Weaver, A. J., and E. S. Sarachik, 1990: On the importance of vertical resolution in certain ocean general circulation models. J. Phys. Oceanogr., 20, 600-609. 
Willoughby, H. E., and P. G. Black, 1996: Hurricane Andrew in Florida: Dynamics of a disaster. Bull. Amer. Meteor. Soc., 77, 543-549.

Xu, M., J.-W. Bao, T. T. Warner, and D. J. Stensrud, 2001: Effect of time step size in MM5 simulations of a mesoscale convective system. Mon. Wea. Rev. 129, $501-516$.

Zhang, D.-L., and R. A. Anthes, 1982: A high-resolution model of the planetary boundary layer-Sensitivity tests and comparisons with SESAME-79 data. J. Appl. Meteor., 21, 1594-1609.

Zhang, D.-L., and J. M. Fritsch, 1988a: Numerical sensitivity experiments of varying model physics on the structure, evolution and dynamics of two mesoscale convective systems. J. Atmos. Sci., 45, 261-293.

Zhang, D.-L., and J. M. Fritsch, 1988b: Numerical simula- tion of the meso- $\beta$ scale structure and evolution of the 1977 Johnstown flood. Part III. Internal gravity waves and the squall line. J. Atmos. Sci., 45, 1252-1268.

Zhang, D.-L., and J. M. Fritsch, 1988c: A numerical investigation of a convectively generated, inertially stable, extratropical warm-core mesovortex over land. Part I: Structure and evolution. Mon. Wea. Rev., 116, 26602687.

Zhang, D.-L., Y. Liu, and M. K. Yau, 2001: A multiscale numerical study of Hurricane Andrew (1992). Part IV:Unbalanced flows. Mon. Wea. Rev., 129, 92-107.

Zhang, D.-L., Y. Liu, and M. K. Yau, 2002: A multiscale numerical study of Hurricane Andrew (1992). Part V: Inner-core thermodynamics. Mon. Wea. Rev., 130, 2745-2763. 